\title{
Communications and Navigation Technologies
}

A White Paper in Response to the Planetary Science and Astrobiology

Decadal Survey 2023 - 2032 Call

Jon Hamkins

Jet Propulsion Laboratory, California Institute of Technology

Jon.Hamkins@.jpl.nasa.gov, 818-354-4764

July 15, 2020

\section{Co-Authors}

Dimitrios Antsos, JPL

James Border, JPL

Gregory Davis, JPL

Leslie Deutsch, JPL

Joseph Lazio, JPL

Jose Velazco, JPL

Acknowledgment. The work presented in this white paper was a collaborative effort carried out at the Jet Propulsion Laboratory, California Institute of Technology, and was sponsored by the National Aeronautics and Space Administration. 


\section{INTRODUCTION}

\subsection{Overview}

Planetary Science missions have both driven and benefited from a steadily increasing data rate capability (Figure 1). Emerging technology can increase data rates by another factor of ten in the next decade. This will enable new science goals of the priority missions of the 2023-2032 Decadal Survey, including orbiters, landers, and subsurface explorers; spacecraft mass ranging from 1 to 3,000 kg; rendezvous and sample return missions; spacecraft constellations; and a variety of destinations including Venus, Mars, ocean worlds, ice giants, and primitive bodies $[1][2][4][6][7][8]$. Specifically, white papers are calling for missions to Titan [9], Triton [10], and Enceladus [11], with instruments that provide ten times the image resolution or capability.

A multi-faceted approach is required to achieve the goal of a factor of ten increase in data rate in the next decade. Next-generation reprogrammable flight radios are needed to incorporate the latest technologies; higher radio frequencies and optical communications are needed to avoid spectrum congestion; better spacecraft amplifiers and antennas are needed to radiate power more efficiently; better channel coding and modulations are needed to make use of limited power and spectrum; better compression is needed to shrink the data sets; better network protocols are needed to ensure reliable transmission through relay orbiters and multiple ground stations; better autonomy is needed for the collection and transmission of scientific data; optical communications flight terminals, pointing architectures, single photon detectors, digital readouts, and ranging are needed to make operational laser communications a reality; and corresponding improvements and upgrades in the Deep Space Network (DSN) ground stations are needed to take advantage of all of these envisioned improvements.

These technologies are described in more detail in the sections below.

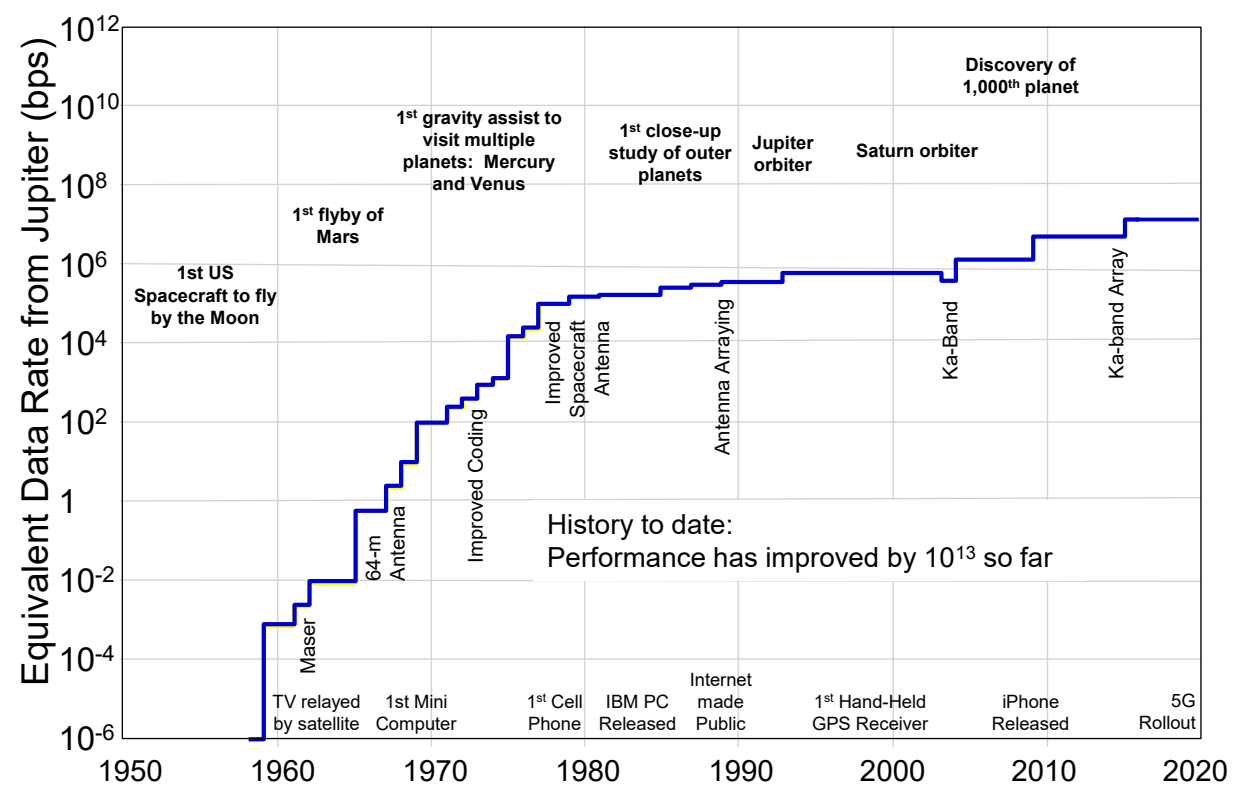

Figure 1: Evolution of data rate capability, normalized to Jupiter distance 


\section{NEEDED COMMUNICATIONS TECHNOLOGIES}

\subsection{Radio frequency (RF) flight transponders}

Next generation RF transponders are needed to provide significant improvements in communications capability (Table 1). The Universal Space Transponder-Deep Space (USTDS, in Figure 2(a)) offers both inflight reprogrammability and data rates up to two orders of magnitude higher than the legacy transponders (Figure 2(c-d)). UST-DS is the next generation deep space transponder, designed for missions such as Mars Sample Return, VERITAS, and Next Mars Orbiter (NeMO). It is a dual-band architecture, supporting UHF, S, X, and Ka-band options, a multitude of modulation types, turbo and LDPC coding, regenerative ranging, and open loop recording.

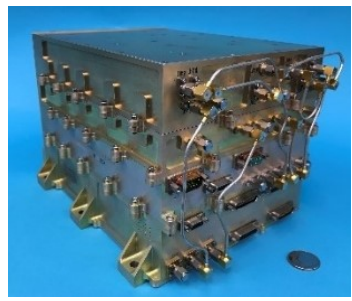

(a) UST-DS

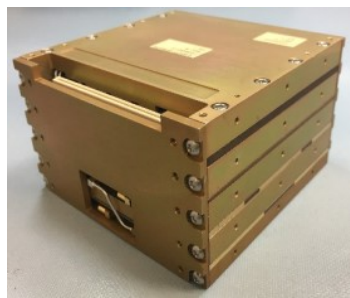

(b) Iris

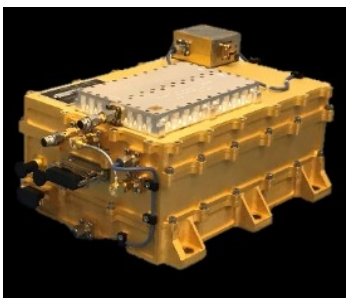

(c) Electra Lite

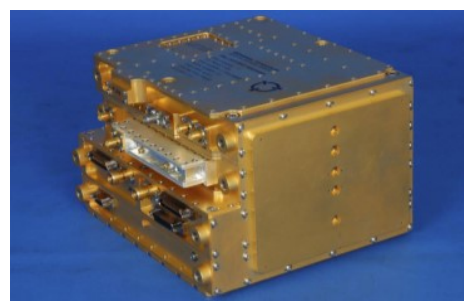

(d) SDST

Figure 2: Next generation and current state of the art transponders

The UST line features a modular hardware architecture with in-flight reprogrammability to provide flexibility and infusion of new technologies, including channel coding, compression, ranging, time correlation and synchronization, radio science, network protocols, electromagnetic interference mitigation, and autonomous radio configuration - many of the technologies mentioned in the other sections of this white paper. UST-Lite is a lower size, weight and power (SWaP) version of UST-DS, repackaged to the SmallSat form factor, yet still supporting up to 300 Msps. UST-KaM is a Ka-band transponder capable of 2 Gsps using higher order modulations, with future upgrades planned to support 4 to 8 Gsps. The Iris (Figure 2(b)) is a flight proven miniature, reprogrammable transponder designed for CubeSats and Class-D missions. It flew to Mars on the MarCO CubeSats launched with InSight. These radios represent the future of planetary mission data transmission.

Table 1: Flight transponder characteristics

\begin{tabular}{cccccc} 
Transponder & $\begin{array}{c}\text { Size } \\
(\mathbf{c c})\end{array}$ & $\begin{array}{c}\text { Weight } \\
(\mathbf{k g})\end{array}$ & $\begin{array}{c}\text { DC Power } \\
(\mathbf{W})\end{array}$ & Band & $\begin{array}{c}\text { Data Rate } \\
\text { (Msps) }\end{array}$ \\
\hline UST-DS & 7500 & 6.3 & 45 & $\mathrm{UHF} / \mathrm{S} / \mathrm{X} / \mathrm{Ka}$ & 300 \\
UST-Lite & 1700 & 1.5 & 28 & $\mathrm{UHF} / \mathrm{S} / \mathrm{X} / \mathrm{Ka}$ & 300 \\
UST-KaM & 5500 & 4.3 & 40 & $\mathrm{Ka}$ & 2,000 \\
Iris & 570 & 0.9 & 14 & $\mathrm{X}$ & 6 \\
SDST (MSL) & 1800 & 2.9 & 16 & $\mathrm{X}$ & 6 \\
Electra & 5200 & 5.1 & 50 & $\mathrm{UHF}$ & 4 \\
Electra Lite & 3400 & 3.0 & 50 & $\mathrm{UHF}$ & 4 \\
\hline
\end{tabular}




\subsection{Optical (laser) communications}

Operational optical communications systems are needed to provide 10 to 100 times higher data rates, compared to equivalent RF systems. Recent progress has been substantial - the Lunar Laser Communications Demonstration (LLCD), Laser Communications Relay Demo (LCRD), and Deep Space Optical Communications (DSOC) technology demonstrations have laid a foundation for future operational systems, and international standards have recently emerged for optical communications at the physical and coding layers [12].

The next decade can truly be the "decade of light" if this technological progress is sustained. DSOC, planned for launch in 2022, plans deep space operation with data rates ranging from $56 \mathrm{Kbps}$ to $267 \mathrm{Mbps}$, utilizing the new high-photon-efficiency signaling standard. Plans are underway to update the DSN RF antennas into RF/optical hybrid antennas (Figure 3), so that both RF and optical signals can be received from a single antenna. The backend receiver electronics will leverage the ground terminals developed for DSOC. Superconducting nanowire single photon detector arrays are being developed to provide the most sensitive performance possible, and with fast $0.1 \mathrm{~ns}$ resolution readouts. New pointing, acquisition, and tracking systems are needed to accurately point the narrow optical laser beam. Noncoherent optical ranging techniques have demonstrated $1 \mathrm{~cm}$ ranging accuracy through the atmosphere, and coherent optical techniques have shown $10 \mathrm{~nm}$ positioning accuracy is possible space-tospace, which enables planetary gravity science and interferometry applications.

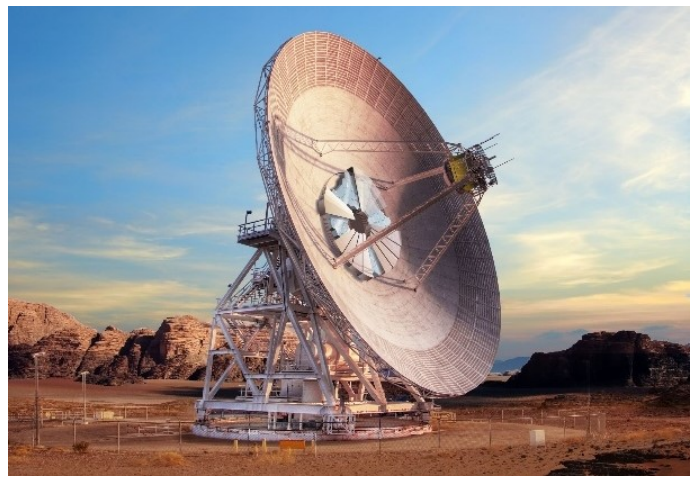

Figure 3: Artist rendering of the RF/optical hybrid antenna

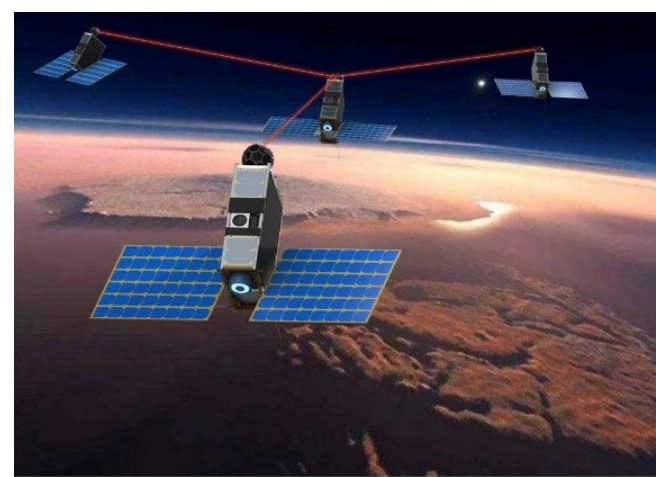

Figure 4: Swarms of spacecraft enabled by omnidirectional optical terminals

Additional advanced technologies will also open up new science opportunities. Omnidirectional optical terminals which provide inter-spacecraft optical links (ISOLs) could be key enablers of new technologies such as swarms and constellations of spacecraft (Figure 4). Swarms of inter-connected spacecraft have the potential to operate autonomously, share data, and act as a large "synthetic" sensor. Swarms, furnished with high resolution sensors for planetary exploration (such as radar, lidar, radiometers, etc.), will demand more bandwidth while still requiring reduced $\mathrm{SWaP}$. This requirement will generate a need for low-power omnidirectional optical transceivers capable of multi-gigabit link rates. In order to be effective, these multi-faceted optical terminals should also provide simultaneous ISOLs, full sky coverage, and high data rates (in excess of $1 \mathrm{Gbps}$ ). 


\subsection{Channel coding and compression}

Infusion of low-density parity-check (LDPC) codes throughout NASA planetary missions can increase science data return by $40 \%$. LDPC is a type of channel coding, sometimes called forward error correction, that has been used for decades to protect data transmission from noise on the channel. Previous missions have used convolutional, Reed-Solomon, and turbo codes. LDPC codes are an international standard of the Consultative Committee for Space Data Systems (CCSDS), and codecs are now available for flight and ground support, but they are not yet widely used for planetary missions. They can be used in conjunction with the autonomous techniques described in Section 2.4 to more than double data return.

New data compression algorithms for specific data types will be needed by the next generation of planetary missions, especially given the size of these data sets. CCSDS is studying and standardizing a low-complexity method to compress synthetic aperture radar (SAR) data, for example, in place of the variety of block-based compression methods currently used. A standard will enable cross support between NASA and other space agencies. Data compression algorithms have already shrunk other types of data by an order of magnitude. The MER and MSL rover cameras, for example, typically took images with 10 bits per pixel and compressed them to 1 bit per pixel with a small manageable loss.

\subsection{Flexibility and autonomy}

Flexibility, adaptability, and autonomy are needed to respond intelligently to dynamic conditions and to provide robust, simpler operations of end-to-end communications systems. Autonomy is an emerging feature of next-generation communications systems. In addition to science autonomy, which automates the collection of scientifically useful data, the communications system itself can be automated. Scientific data return can be more than doubled by a system which can vary its data rate, channel code, or modulation type in response to dynamic link conditions such as the changing range of an orbiter seen by a rover. This is called variable coded modulation (VCM) and is an emerging space standard, but not yet widely deployed. If these dynamic changes can be automatically detected, then adaptive coded modulation (ACM) can increase data volume further, while simplifying operations. In a fully automated set up, a PI could request a scientific observation from her desktop and have the data be captured and returned, seamlessly traversing one or more deep space assets, the Deep Space Network, Multimission Ground Systems and Services (MGSS), and the Internet.

\subsection{Power amplifiers}

Advanced technology is needed to develop the low-SWaP, efficient, high power amplifiers demanded by planetary exploration. Achievable communication data rate is proportional to the transmitted RF power, which means that improvements in amplifier efficiency directly translate into higher aggregate mission data return. Improvements can also be made on mass and volume, improving the overall performance of the communications system.

The current challenge is to develop a Ka-Band solid-state power amplifier (SSPA) to match or exceed the efficiency of a traveling wave tube amplifier (TWTA) at comparable power levels. NASA has been transitioning to Ka-Band over the last decade because the higher frequency of Ka-band has a greater than a factor of two power advantage over X-band transmission, and 
there is more bandwidth available at the higher frequency. However, building high power, high efficiency power amplifiers is more challenging at the higher frequencies.

In the state of the art, Ka-Band SSPAs are mostly based on transistors built on Gallium Arsenide or Indium Phosphide semiconductor substrates, and TWTAs are based on vacuum tube technologies. SSPAs currently in flight are typically small (hundreds of grams and milliliters) but have low output power (under $10 \mathrm{~W}$ ) and efficiency (typically under 20\%). TWTAs have much higher output power (up to about $500 \mathrm{~W}$ commercially available) and efficiency (up to about 50\%), but are much bigger (a few liters) and much more massive (several $\mathrm{kg}$ ), and they require high-voltage (of the order of $10 \mathrm{kV}$ ) power systems.

Investment is needed in SSPAs, which can consist of a single, very small Monolithic Microwave Integrated Circuit (MMIC), typically a few millimeters on the side, or several MMICs packaged together, the output power of which is combined to a single output connector. A state-of-the-art commercial, flight qualified SSPA has a output power of $22 \mathrm{~W}$ at an efficiency of $25 \%$, when driven to saturation. Missions to the outer planets or missions having instruments that generate high quantities of data may require higher RF communication power. For example, the Kepler mission flew a 40\%-efficient $35 \mathrm{~W}$ Ka-Band TWTA. On a single MMIC, performance can be limited, but by combining the output power of multiple MMICs off-chip, as part of a "hybrid" (both on-chip and off-chip) architecture, higher output power and efficiency can be achieved.

\subsection{Deployable mesh reflectors}

Large, deployable mesh reflectors are needed as part of the solution to provide increased data rate by an order of magnitude in the next decade. These are now common in the Earth-orbiting environment, but not in deep space. For example, the Soil Moisture Active Passive (SMAP) Earth-orbiting mission recently flew a 6-m diameter, rotationally scanning deployable/ unfurlable mesh main antenna reflector. This reflector operates at $\mathrm{L}$ band $(1 \mathrm{GHz}-2 \mathrm{GHz})$ with a wavelength-to-diameter (WDR) ratio of 30. The to-be-flown NASA-ISRO SAR mission (NISAR) will carry a 12-m diameter deployable L- and S-band radar antenna with a WDR = 130. The highest gain antenna flown by NASA, as defined by the WDR ratio, remains the 5m Ku-band (13 GHz-15 GHz) deployable mesh reflector built by the Harris Corporation and used on the Tracking Data and Relay Satellite (TDRS) A-G satellites (in Figure 5). These antennas have $\mathrm{WDR} \approx 250$ and are all passive in the sense that, once deployed, no mechanisms are required to maintain the figure of the antenna.

Deployable mesh reflectors are routinely available from industry with apertures as large as 22 $\mathrm{m}$, but none of them have exceeded the TDRS benchmark WDR ratio until now: new developments by both Harris and NGAS have pushed the state of the art to WDR $\approx 500$ with 5-m-class Ka-band apertures. The new state of the art is by no means "routine," and no results from on-orbit deployment or operation of Ka-band mesh reflectors have yet been made public. Nonetheless, both vendors are vigorously marketing the new capability with convincing ground demonstration hardware and claim to be working with customers towards future flight projects. 


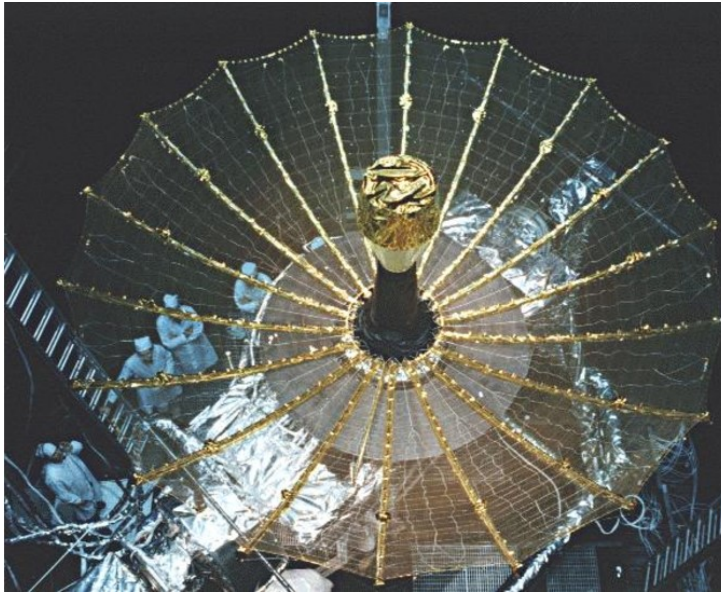

Figure 5: The 5-m diameter deployable Ku-band TDRS mesh antenna

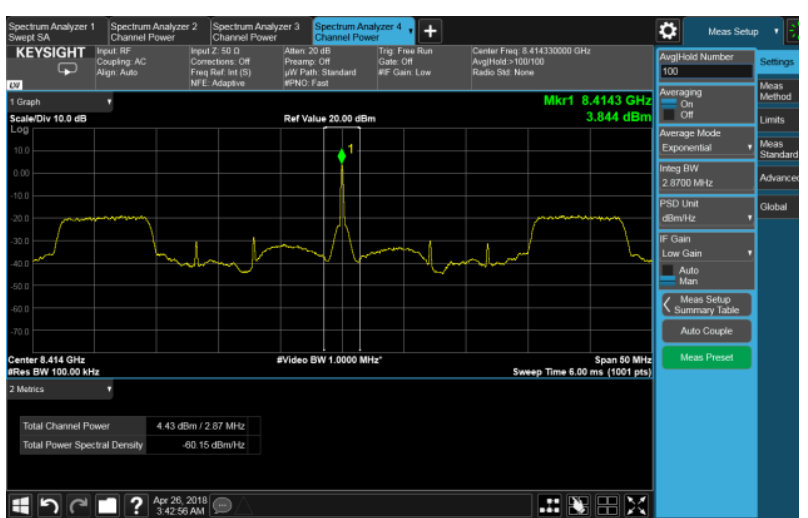

Figure 6: X-band DOR sidebands spread by a PN code with pulse shaping

\subsection{Navigation}

Advanced navigation technology is needed to meet the demand of planetary science missions for more precise targeting for landings, flybys, or scientific observation. Now, enhanced capabilities are being made possible by the newer generation of software defined radios. Both Iris and UST support pseudo-noise (PN) regenerative ranging. This is particularly useful for SmallSat missions with limited telecom performance. It also paves the way for a next generation ranging in which timing is obtained directly from telemetry signals, which allows telemetry at higher bit rates while still providing high accuracy ranging.

Both Iris and UST also support PN spreading of differential one-way ranging (DOR) tones. The Delta-DOR technique measures the angle between a spacecraft and a quasar to provide high accuracy plane-of-sky coordinates. The PN spreading matches the spacecraft spectrum to the quasar spectrum and allows common mode cancellation of instrumental error. Work in progress should provide accuracy at the $0.5 \mathrm{nrad}$ level at Ka-band. High accuracy is applicable for targeting of planetary encounters and also for positioning of astrometry missions or constellations whose science depends on knowing the precise state of the observing spacecraft. Figure 6 shows the Iris DOR spectrum. The quasar spectrum is flat across the full band. The spacecraft spectrum has been flattened across frequency ranges that will be recorded to obtain the differential angle measurement.

Precise Doppler has long contributed to Radio Science investigations. For the first time, ranging with $10 \mathrm{~cm}$ accuracy is also available to contribute to Radio Science investigations. The DSN has developed the capability to uplink a 24 Mcps range code at Ka-band. The ESA mission BepiColombo carries a transponder to regenerate this high chip rate PN code as part of its Radio Science instrument. Multiple frequency links are required to keep accuracy at the $10 \mathrm{~cm}$ level through solar conjunctions. Improved ranging contributes to tests of general relativity and can also improve ephemerides of solar system bodies. 


\subsection{Delay/Disruption Tolerant Networking (DTN)}

Investment is needed to provide the full suite of DTN capabilities, security enhancements, and enhanced performance needed by planetary exploration. DTN is a suite of standard protocols that route bundles of data end-to-end through network nodes even in situations that involve delays, disconnections, and data rate mismatches. It automates reliable communications through multiple user spacecraft, relays, ground stations, and science facilities.

The development and deployment of DTN is critical for planetary and astrobiology science missions as they become numerous, networked, or automated, and it eases the operational burden while providing maximum flexibility for PIs to request scientific data from their instruments. DTN can dynamically switch communication paths when intermediate nodes appear or disappear, which happens as relays come into and out of view and ground stations handover as the Earth rotates. Outages or errors are detected and data is retransmitted without humans in the loop. NASA uses DTN on the International Space Station, and has plans to support the KPLO mission (up to $20 \mathrm{Mbps}$ ), PACE (up to $150 \mathrm{Mbps}$ ), and LCRD (1 to 100 Gbps) in 2021-2022. DTN can be implemented on the radios described in Section 2.1.

\section{CONCLUSIONS AND RECOMMENDATIONS}

The science goals and priority missions of the 2023-2032 Decadal Survey will benefit from improved communications and navigation capabilities. Increasing data rate capability by a factor of ten in the next decade is within reach, with investments in key communications technologies, including (a) next generation reprogrammable flight transponders designed for orbiters, landers, and specialty applications, operating at Ka-band, and with reduced SWaP; (b) optical communications technologies including flight and ground terminals, detectors, readouts, standards, pointing, ranging, and omnidirectional terminals; (c) infusion of LDPC codes on planetary missions and compression for SAR and other targeted data types; (d) variable data rates and adaptive coded modulation to autonomously adapt to link conditions; (e) solid state power amplifiers with improved efficiency and power output; (f) deployable antennas for deep space; (g) new two-way ranging, Delta-DOR, and Doppler techniques; and (h) infusion of DTN throughout the space and ground networking infrastructure.

\section{References}

[1] "Advanced Communication and Navigation (C\&N)," NASA STMD roadmap, 2020

[2] JPL 2018 Strategic Implementation Plan. https://tinyurl.com/y7lsv56u

[3] JPL: Strategic Technologies 2019. https://tinyurl.com/y8v62g8p

[4] Planetary Science Decadal Survey 2013-2022. https:/tinyurl.com/y7c9rvnj

[5] B. Giovannoni, et al., white paper, Planetary/Astrobiological Decadal Survey 2023-2032

[6] NASA 2017 Ice Giant Study Report: https://www.lpi.usra.edu/icegiants/mission_study/

[7] Roadmaps to Ocean Worlds (ROW) study report: https://www.lpi.usra.edu/opag/ROW/

[8] OPAG Science Goals document, https://www.lpi.usra.edu/opag/goals-08-28-19.pdf

[9] J. Barnes, et al., white paper, Planetary/Astrobiological Decadal Survey 2023-2032

[10]C. Hansen, et al., white paper, Planetary/Astrobiological Decadal Survey 2023-2032

[11] M. Cable, et al., white paper, Planetary/Astrobiological Decadal Survey 2023-2032

[12] CCSDS Optical Communications standards, 141.0-B-1, 142.0-B-1 